\title{
Personality predicts working-memory-related activation in the caudal anterior cingulate cortex
}

\author{
JEREMY R. GRAY and TODD S. BRAVER \\ Washington University, St. Louis, Missouri
}

\begin{abstract}
Behavioral studies suggest that two affective dimensions of personality are associated with working memory (WM) function. WM load is known to modulate neural activity in the caudal anterior cingulate cortex (ACC), a brain region critical for the cognitive control of behavior. On this basis, we hypothesized that neural activity in the caudal ACC during a WM task should be associated with personality: correlated negatively with behavioral approach sensitivity (BAS) and positively with behavioral inhibition sensitivity (BIS). Using functional magnetic resonance imaging, we measured brain activity in 14 participants performing a three-back WM task. Higher self-reported BAS predicted better WM performance $(r=.27)$ and lower WM-related activation in the caudal ACC $(r=-.84)$, suggesting personality differences in cognitive control. The data bolster approach-withdrawal (action control) theories of personality and suggest refinements to the dominant views of ACC and personality.
\end{abstract}

The dynamic interaction between affective and cognitive processes has long fascinated psychologists and other close observers of human nature (Bless \& Schwarz, 1999; Easterbrook, 1959; Humphreys \& Revelle, 1984; Isen, 1993; Leon \& Revelle, 1985; Metcalfe \& Mischel, 1999; Simon, 1967; Yerkes \& Dodson, 1908). Recently, the topic has become increasingly tractable in terms of neurobiology as well (Damasio, 1994; Simpson, Snyder, Gusnard, $\&$ Raichle, 2001). In this context, the anterior cingulate cortex (ACC) is a particularly intriguing neuroanatomical structure. On the one hand, "an important guiding principle about ACC function is that cognitive and emotional information are processed separately" (Bush, Luu, \& Posner, 2000, pp. 215-216). On the other hand, the fact that cognitive and emotional functions are both mediated by the same region of the brain hints at a possible basis for their interaction (Bush et al., 2000; Luu, Colins, \& Tucker, 2000; Simpson et al., 2001).

To further investigate the relationship between emotion and cognitive control in terms of ACC function, we focused on the possibility of associations between two affective personality traits and ACC activity during a working memory (WM) task. Two major dimensions of personality are strongly affective, being associated with stable individual

This work was supported by the National Science Foundation, BCS 0001908, and the McDonnell Center for Higher Brain Function, Washington University. We thank John M. Zelenski, Deanna M. Barch, Kathleen McDermott, Marcus E. Raichle, Randy J. Larsen, Jesse Prinz, and the other members of the emotion-cognition reading group at Washington University for specific comments and many productive discussions that have helped shape our thoughts on these issues. Correspondence concerning this article should be be addressed to J. R. Gray or T. S. Braver, Department of Psychology, Campus Box 1125, Washington University, St. Louis, MO 63130 (email: jeremy_gray@ post.harvard.edu or tbraver@artsci.wustl.edu). differences in arousal (H. J. Eysenck \& M. W. Eysenck, 1985; Revelle, 1993) and ongoing mood and emotional reactivity (Costa \& McCrae, 1980; Gross, Sutton, \& Ketelaar, 1998; Larsen \& Ketelaar, 1991; Watson \& Tellegen, 1985). Interestingly, affective personality variables are also associated with cognitive differences, including differences in WM (M. W. Eysenck \& Calvo, 1992; Lieberman \& Rosenthal, 2001). WM refers to the ability to actively maintain and manipulate information in the mind (Baddeley, 1986; Miyake \& Shah, 1999) and is a key aspect of higher cognitive functions. In Baddeley's model, WM consists of two modality-specific buffers for the active maintenance of phonological and visuospatial information and a central executive component for manipulating that information. In neuroimaging studies, WM tasks robustly activate the ACC (Cabeza \& Nyberg, 2000). On this basis, it is possible that affective personality variables should predict ACC activity during a WM task. To explain this hypothesis, we first provide more background on both the ACC and personality.

\section{Anterior Cingulate Cortex}

The ACC is an important neural structure in the medial prefrontal cortex and is involved in the executive control of behavior (Bush et al., 2000; Carter, Botvinick, \& Cohen, 2000; Devinsky, Morrell, \& Vogt, 1995; Posner \& Petersen, 1990). The caudal region of the ACC is activated during diverse challenging, but not easy, cognitive tasks (Paus, Koski, Caramanos, \& Westbury, 1998). The caudal ACC is sensitive to the demand for control and is part of a larger network subserving cognitive control function. The activation of the caudal ACC during WM tasks appears to signal the increased demands on cognitive control when WM load is high. The more specific contribution of the caudal ACC to the overall function of this network is a topic of active investigation(e.g., Botvinick, Braver, Barch, Carter, \& Cohen, 2001; Bush et al., 2000; Carter et al., 
1998; Miltner, Braun, \& Coles, 1997; Paus et al., 1998; Posner \& DiGirolamo, 1998).

A largely separate literature has focused on affective processing in other subregions of the ACC. The rostral and subgenual ACC appear to be particularly sensitive to emotion (see Bush et al., 2000, for a review). These subregions are engaged during the experience or anticipation of painful stimuli (Derbyshire, Vogt, \& Jones, 1998; Johansen, Fields, \& Manning, 2001). Depression seems to be related to abnormally low levels of activity in these regions (Drevets et al., 1997; Mayberg et al., 1997). Such findings have lead to a model of the ACC in which the rostral and subgenual regions mediate affective function, whereas the caudal regions mediate cognitive function (Bush et al., 2000; Devinsky et al., 1995; Whalen et al., 1998). An emotional Stroop task activated the rostral ACC, whereas a neutral version activated the caudal ACC (Whalen et al., 1998), a double dissociation providing direct support for this model. Demanding cognitive tasks consistently deactivate the rostral and subgenual areas (Shulman et al., 1997), also supporting the model. Finally, individual differences in rostral and subgenual ACC activity during a resting state have been related to selfreported affective personality variables. Before we consider such data in more detail, we will provide slightly more background on personality.

\section{Personality}

Personality refers to stable individual differences, especially differences in temperament, disposition, or character (Funder, 2001; Larsen \& Buss, 2002). Personality is often assessed by using self-report questionnaires in which participants indicate how strongly various statements characterize them. In factor analyses of such self-report data in healthy participants, between three and seven major dimensions of personality typically emerge. There is wide agreement that two of the strongest and most consistent dimensions are affective in nature (Cloninger, Svrakic, \& Przybeck, 1993; Costa \& McCrae, 1980; Depue \& Collins, 1999; H. J. Eysenck, 1967; J. A. Gray, 1970; Larsen \& Buss, 2002; Larsen \& Ketelaar, 1991; Zelenski \& Larsen, 1999).

In one theory of personality, the two affective dimensions are referred to as extraversion and neuroticism (H. J. Eysenck, 1967; H. J. Eysenck \& M. W. Eysenck, 1985). Extraversion is associated with positive affect, gregariousness, and arousability, whereas neuroticism is associated with unstable and especially negative affect (e.g., depression, anxiety). These two traits are strongly associated with susceptibility to pleasant and unpleasant emotional states, respectively (Costa \& McCrae, 1980; Gross et al., 1998; Larsen \& Ketelaar, 1991; Watson \& Tellegen, 1985; Zelenski \& Larsen, 1999). In another theory of personality, there are also two affective personality dimensions, termed impulsivity (sensitivity to cues of reward) and anxiety (sensitivity to cues of punishment; J. A. Gray, $1970,1982,1994)$. The anxiety dimension reflects individual differences in the strength of a behavioral inhibition system (BIS) sensitive to threat. The impulsivity di- mension reflects individual differences in the strength of a behavioral approach (or activation) system (BAS) sensitive to reward. Although there are differences between the BIS-BAS and the neuroticism-extraversion conceptions of personality, a recent integrative review suggested that the commonalities are more interesting and perhaps more fundamental than the differences (Carver, Sutton, $\&$ Scheier, 2000). Factor analysis of various self-report scales within the same participants suggests a similar interpretation (Zelenski \& Larsen, 1999).

Although not the dominant view, Carver et al.'s (2000) theory of personality is particularly noteworthy for its aim: to integrate personality with processes related to the control of behavior. By emphasizing approach- and withdrawal-related motivation as core defining features of extraversion and neuroticism, their theory suggests a functional, process-oriented basis of personality. That is, the two major affective dimensions reflect individual differences in two systems for action control (approach, withdrawal). A control system perspective suggests that personality might be related to cognitive aspects of control, as we will discuss below.

Given our interest in personality and cognitive control, we focused on the two aspects of personality that Carver et al. (2000) highlight as most germane to action control: BIS and BAS. Our previous work also leads us to expect on empirical grounds that BIS and BAS might be related to differences in cognitive control. Self-reported BIS and BAS (using scales developed by Carver \& White, 1994) predicted both affective reactivity and the influence of induced emotion on verbal versus nonverbal WM performance (J. R. Gray, 2001). In contrast, self-report measures of extraversion and neuroticism (using the EPQ-R; S. B. G. Eysenck, H. A. Eysenck, \& Barrett, 1985) did not predict performance in the same participants. The different scales probably assess different aspects or subfactors of personality (Carver \& White, 1994). The EPQ-R assesses mainly the sociability subfactor of extraversion, whereas BAS assesses the strength of approach motivation, particularly in response to specific events.

Other work has shown that individualdifferences in BIS and BAS predict differences in prefrontal hemispheric asymmetries in resting brain activity (Harmon-Jones \& Allen, 1997; Sutton \& Davidson, 1997). High-BAS participants had greater left frontal neural activity at rest, whereas high-BIS participants had greater right frontal activity at rest. This is consistent with considerable work showing that these asymmetries are strongly affective and related to approach-withdrawal motivation specifically (Davidson, 1995; Harmon-Jones \& Sigelman, 2001). The asymmetry may reflect activity in the lateral prefrontal cortex specifically (J. R. Gray, Braver, \& Raichle, in press), another brain region critical for WM and cognitive control (Braver et al., 1997; D'Esposito et al., 1995; Duncan et al., 2000; Goldman-Rakic, 1987; Smith \& Jonides, 1999). In contrast, in Sutton and Davidson's data set, measures of dispositionally positive or negative affect (Watson, Clark, \& Tellegen, 1988) did not predict asymmetric 
brain activity in the same participants. In another study, extremely sociable versus extremely shy women differed in their resting prefrontal asymmetries (Schmidt, 1999). The sociable group had greater left prefrontal activity, and the shy group had greater right prefrontal activity. These data support the idea that extraversion and sociability are related to approach motivation (BAS) and social anxiety and shyness are related to withdrawal motivation (BIS), consistent with Carver et al.'s (2000) action control account of personality.

\section{Personality and Working Memory}

Several behavioral studies suggest a relationship between WM and the affective dimensions of personality (M. W. Eysenck \& Calvo, 1992; Humphreys \& Revelle, 1984; Lieberman \& Rosenthal, 2001; Mathews, Davies, \& Lees, 1990; Revelle, 1993).

Higher extraversion is associated with better WM performance (Lieberman, 2000; Lieberman \& Rosenthal, 2001). Lieberman and Rosenthal reasoned that extraverts' greater fluency in social situations might be due to better WM, rather than to expertise at social information processing or greater motivation. Nonverbal decoding (e.g., accurately inferring other peoples' affect from their facial expression, voice prosody, posture, and so on) is important in social interaction, but surprisingly, a meta-analysis found no evidence to suggest that extraverts perform better than introverts on such tasks in the laboratory. However, social interaction requires not just nonverbal decoding, but nonverbal decoding while simultaneously maintaining a conversation. Extraverted individuals' advantage may be in coordinating simultaneous social tasks (e.g., nonverbal decoding, conversation maintenance), and such multitasking is likely to require central executive processes. Lieberman and Rosenthal's WM account of extraversion was consistently upheld across experiments: An extravert advantage was evident only when multitasking was required, with no difference on nonverbal decoding tasks in isolation. A central executive locus of the trait difference was suggested on the basis of performance on standard laboratory tasks of WM (verbal $n$-back tasks), by showing a stronger relationship at higher levels of load.

Trait anxiety is associated with worse performance on diverse demanding cognitive tasks that are likely to require WM (Darke, 1988; M. W. Eysenck, 1979). Although such behavioral impairments are not always found, many of the null results can be explained if trait anxiety is associated with differences in processing efficiency, rather than with differences in the level of performance (M. W. Eysenck \& Calvo, 1992). That is, anxious individuals are held to need to exert greater mental effort in order to achieve the same level of behavioral performance-that is, they are less efficient but can often compensate by working harder. It is only when they are unable to compensate by exerting greater effort that their performance should suffer.

The exertion of compensatory effort can be difficult to infer solely on the basis of behavioral performance. How- ever, physiological measures can provide a measure of effort not confounded with performance (M. W. Eysenck $\&$ Calvo, 1992). Although the processing efficiency hypothesis was developed only with reference to trait anxiety, we suggest that an analogous account may also hold for extraversion, BAS, or both: Introverted individuals (low extraversion, low BAS) might not always show a behavioral deficit on tasks requiring cognitive control, but differences in compensatory effort should be evident physiologically in such circumstances. The caudal ACC is a natural candidate region in which such differences may be observed.

\section{Personality and the Anterior Cingulate}

Several neuroimaging studies suggest associations of personality measures with ACC activity during a resting state. Some have found such associations the in rostralsubgenual ACC, and not in the caudal ACC (Johnson et al., 1999; Zald, Mattson, \& Pardo, 2002), and other studies have found associations in the ACC but could not further localize subregions of the ACC (Ebmeier et al., 1994). Activity in the posterior part of the rostral ACC differed by personality in response to viewing emotional images (Canli et al., 2001). Thus, during resting or emotional conditions, previous work suggests relations between personality and the rostral and subgenual, but not the caudal, ACC - in agreement with models of regional specialization for cognitive versus affective function within the ACC (Bush et al., 2000; Devinsky et al., 1995).

Neuroimaging measures provide a way to test predictions of personality theories that posit differences not only in resting state activity, but also in processing efficiency and compensatory effort. Because numerous group-level studies consistently show that activity in the caudal ACC indicates the demand for control, we predicted that individual differences in activity should be evident in the ACC during a cognitive challenge and should be related to personality: Cognitivelyefficient individuals (high BAS, low BIS) should have lower task-related activity.

In terms of ACC function, a straightforward extrapolation from cognitive-personality theories is that personality should influence the caudal (cognitive) as well as the rostral-subgenual (affective) subregions. However, there has been little investigation of this issue. A suggestive study showed that ACC activity during errors in cognitive task performance was associated with neuroticism, as assessed by event-related potentials (ERPs; Luu et al., 2000). The ERP activity was localized to the medial frontal cortex, suggesting that the ACC was the source of the signal, but was not further localized to cognitive or affective subregions (see Luu, Tucker, Derryberry, Reed, \& Poulsen, in press).

To our knowledge, no study has manipulated both cognitive and emotional demands to examine associations between personality variables and ACC activity, using a brain-imaging method with high spatial resolution. From the perspective of functional neuroanatomy alone, an association of personality with caudal (cognitive) ACC ac- 
tivity would be a strongly counterintuitive prediction. On the basis of the considerable evidence for an anatomical segregation of affective and cognitive functions within the ACC, rostral-subgenual (affective) regions should be sensitive to personality, whereas caudal (cognitive) regions should not. Alternatively, extrapolating from the literature on personality-cognition interactions, a relationship should hold between personality and WM-related activity in the caudal ACC (given the additional assumption that the caudal ACC is sensitive to the demand for cognitive control, which is amply documented). That is, personality should be associated with differences in the demand for cognitive control, and such variation should be reflected in caudal ACC activity during a WM task. Such a result could have important implications for theories of both ACC function and personality-cognition relations, suggesting a need to refine the dominant views.

The strongest kind of result would involve not just an association, but one in which individual differences in behavioral performance cannot explain differences in ACC activity. Behavior represents the output of the whole system, including nonspecific effects (e.g., low motivation to engage a difficult task), as well as effects of components of the system that might compensate for the underfunctioning of other components. Behavior is thus a relatively insensitive or nonspecific measure. In contrast, ACC activity is well established as reflecting the demand for (or exertion of) control relatively purely. Nonspecific differences (e.g., in task motivation) could lead to a less interesting relation between personality variables and ACC activity, but such nonspecific differences would manifest in behavioral performance as well. Thus, finding a correlation between personality and ACC activity during a cognitive challenge would be a stronger finding if it could not be explained by differences in performance.

Although our primary interest was in personality, emotional state was a potentially important variable. Affective dimensions of personality are reliably associated with differences in ongoing mood. This suggests that emotional states could potentially mediate an association between personality and cognitive activation. Another possibility is that emotional states might interact with and influence a relation between personality and cognition. Induced emotional states are likely to act as a trait-related challenge or provocation and so might moderate an association, making it more or less pronounced.

Thus, we tested for associations of two affective personality variables (BIS, BAS) with neural activity in the ACC in the caudal (cognitive) subregion related to WM task performance. We also tested whether induced emotional states moderate such trait relations.

\section{METHOD}

Participants completed self-report personality questionnaires and then performed a WM task after a preceding emotional video had ended. The fMRI participants $(n=14)$ were scanned as they performed the WM tasks. Given our small fMRI sample, we were particularly interested in relations between personality and brain ac- tivity that reached significance despite low statistical power. An additional group ( $n=115)$ was tested offline to assess the relationship between personality and behavioral performance with greater statistical power (total $n=129$ ).

\section{Participants and Procedure}

Neurologically normal, right-handed participants ( $n=14,6$ males, 19-27 years of age) gave informed consent and were paid \$25 an hour for participation in the fMRI scan. An additional group of 115 participants completed the same behavioral protocol (trait measures, emotion inductions, and three-back tasks in the same design).

Trait measures. To assess the affective dimensions of personality, we used the BIS and BAS scales (Carver \& White, 1994). These measures have good psychometric properties (normality of distribution, test-retest reliability, convergent and discriminant validity) and have been able to predict relevant outcome measures in our previous work.

\section{Cognitive State Manipulation}

To manipulate the demand for cognitive control, the participants performed an $n$-back WM task (three-back) that alternated with periods of resting fixation. The $n$-back task involves several cognitive components, not all of which require control (e.g., visual perception of the stimuli is critical for performance but is largely an automatic process). However, cognitive control can be readily inferred on the basis of functional neuroanatomy. That is, to infer specificity of the trait relation to cognitive control, our strategy was (1) to document personality differences in WM function by showing a relationship between personality and behavioral performance, replicating previous work, and (2) to rely on a wealth of prior evidence suggesting that caudal ACC activity indexes the demand for (or exertion of) control.

The participants performed a standard WM task, a version of the three-back continuous performance test. The task instructions were to press the target button if the stimulus currently on the screen was the same as the one seen three trials previously (i.e., three back) and the nontarget button for any other stimulus. All the stimuli were taken from a previous fMRI study of the $n$-back task (Braver, Barch, Kelley, et al., 2001) and were either all words or all faces for a given scanning run. In the word three-back conditions, the participants viewed concrete English nouns, one to four syllables in length. In the face condition, they saw unfamiliar male and female faces intermixed. The two stimulus types were included as part of another experiment focusing on interactions between emotional state and the domain of WM (J. R. Gray et al., in press). In the fMRI analyses reported here, stimulus type was largely irrelevant, and so we collapsed over it (yet we examined it as a factor of interest in the large behavioral sample). During each scanning run, the participants performed the three-back task in short task blocks $(40 \mathrm{sec}=$ 16 trials, each trial being $2.5 \mathrm{sec}$ ) that alternated with short blocks of resting fixation $(25 \mathrm{sec})$. Each run consisted of four cycles of the task and fixation blocks. Performance on each trial was scored for response time (RT) and accuracy by the computer.

Emotional state manipulation. To induce emotional states, short videos (pleasant-approach, neutral, or unpleasant-withdrawal) were shown just prior to each WM task and scan. There were six different videos for the emotion inductions (two versions of three emotion types). The videos were the same as those used elsewhere (J. R. Gray, 2001): 9- to 10-min selections from comedy (pleasant, amusing), documentary (neutral, calm), or horror (unpleasant, anxiety) films. After each scanning run, the participants used the response buttons to rate how they had felt after the last video just prior to the scan, from zero (not at all) to four (very much) for each of six emotion terms presented in random order (calm, amused, anxious, energetic, fatigued, and gloomy).

Procedure. Prior to the scan session, the participants had $30 \mathrm{fa}-$ miliarization trials with the $n$-back task. Inside the scanner, the participants had structural scans, followed by six functional scans in a 
$3 \times 2$ factorial design: 3 (emotion type: pleasant, neutral, or unpleasant) $\times 2$ (stimulus type: faces or words). The order was counterbalanced across participants. For each functional run, the $n$-back task and scan began as soon as possible after the end of each video.

The tasks were presented using PsyScope (Cohen, MacWhinney, Flatt, \& Provost, 1993) on an Apple Macintosh computer. The videos were presented digitally (QuickTime format, resolution $320 \times$ 240). All visual stimuli were projected onto a screen at the head of the bore. Headphones dampened scanner noise, enabled communication with participants, and were used for the audio portion of the videos. The scanner room was dark during the videos and functional scans.

\section{Image Acquisition and Analysis}

Images were acquired on a Siemens 1.5 Tesla Vision System (Erlangen, Germany) with a standard circularly polarized head coil. A pillow and tape were used to minimize head movement. Structural images were acquired by using a high resolution $(1.25 \times 1 \times 1 \mathrm{~mm})$ sagittal 3-D MP-RAGE (Mugler \& Brookeman, 1990) T1-weighted sequence $\left(\mathrm{TR}=9.7 \mathrm{msec}, \mathrm{TE}=4 \mathrm{msec}\right.$, flip $\left.=12^{\circ}, \mathrm{TI}=300 \mathrm{msec}\right)$. Functional images were acquired by using an asymmetric spin-echo echo-planar sequence $\left(\mathrm{TR}=2,500 \mathrm{msec}, \mathrm{TE}=50 \mathrm{msec}\right.$, flip $\left.=90^{\circ}\right)$. During each functional scanning run, 108 sets of 16 contiguous, $8-\mathrm{mm}$ thick axial images were acquired parallel to the anterior-posterior commissure plane $(3.75 \times 3.75 \mathrm{~mm}$ in-plane resolution), allowing complete brain coverage at a high signal-to-noise ratio (Conturo et al., 1996).

Following movement correction, all functional images were scaled to achieve a whole-brain mode value of 1,000 (used in place of a mean because of its reduced sensitivity to variation in brain margin definition) for each scanning run (to reduce the effect of scanner drift or instability). Functional images were then resampled into 3-mm isotropic voxels, transformed into standardized atlas space (Talairach \& Tournoux, 1988), and smoothed with a Gaussian filter ( $8 \mathrm{~mm}$ full width at half maximum). All coordinates are given in Talairach space. For statistical analyses, the participants served as the random effect.

Technical problems resulted in the loss of fMRI data for one scan (face-unpleasant condition). This participant was included in all analyses for which complete data were available.

\section{RESULTS}

\section{Behavioral and Self-Report Measures}

Cognitive manipulation. Each participant's behavioral performance was averaged across the six scans. The group mean $(S D)$ for RTs was $1,076 \mathrm{msec}(200)$; the group mean for accuracy was $88 \%$ correct $(8 \%)$. Thus, the tasks imposed a substantial cognitive load, and yet performance was far above chance levels.

Emotional manipulation. The ratings verified that the emotion induction procedure elicited the intended emotional states and that the nonneutral states were arousing and matched for arousal level. We compared how strongly the participants rated feeling the intended emotion after the appropriate video, as compared with the other videos (e.g., the average rating of anxious after the two unpleasant videos minus the average of anxious after the other four). The emotion induction produced selective increases in selfreports of anxious after the unpleasant videos, calm after the neutral videos, and amused after the pleasant videos [all $\left.t_{\mathrm{s}}(13)>3.70, p \mathrm{~s}<.004\right]$. Arousal was assessed in a similar manner. Mean ratings of energetic were significantly greater after either the pleasant or the unpleasant videos than after the neutral videos [ $t \mathrm{~s}(13)>2.38, p \mathrm{~s}<.03$ ], yet did not differ between pleasant and unpleasant videos $[t(13)<1]$.

Personality. In the 14 participants who were scanned, BIS scores ranged from 15 to 27 (mean, 20.7; SD, 3.7), BAS scores ranged from 28 to 49 (mean, 39.9; $S D, 6.4$ ). BIS and BAS were not correlated $(r=.04)$. Although extremely low BIS participants were underrepresented, these ranges are otherwise representative of the larger sample $(n=115$; BIS, range of 10-28, mean $=20.8, S D=4.4$; BAS, range of 26-52, mean $=41.2, S D=5.0$; BIS-BAS correlation, $r=-.15$ ). One fMRI participant was at the extreme high end on BIS and the extreme low end on BAS. Although we had no reason to doubt the validity of the data point, we performed all analyses by both including and excluding this participant. There were only slight differences between these analyses, our conclusions were not affected, and so we report the analyses that included this participant.

We tested whether personality predicted behavioral performance in all 129 participants. Following Lieberman and Rosenthal's (2001) analysis for extraversion, trait groups were defined on the basis of mean splits, creating two BAS groups (high BAS, low BAS) and two BIS groups (high BIS, low BIS). To enable a test of whether associations between personality and performance held equally for verbal and nonverbal WM, the type of stimulus in the $n$-back task (word, face) was retained as a factor of interest. Analyses of variance (ANOVAs) were computed for BIS and BAS separately. Each ANOVA model had a 2 (trait group: high, low) $\times 2$ (stimulus type: word, face) factorial design. The dependent measure was behavioral performance, with separate analyses for accuracy $\left(d^{\prime}\right)$ and RT. Group means and effect sizes are reported in Table 1. For $d^{\prime}$, the high-BAS group had better performance (higher $d^{\prime}$ ) than the low-

Table 1

Personality Predicts Behavioral Performance

\begin{tabular}{lccccccc}
\hline & \multicolumn{3}{c}{$n=129$} & & \multicolumn{3}{c}{$n=14$ (scanned) } \\
\cline { 2 - 3 } & $n$ & $d^{\prime}$ & $\mathrm{RT}$ & & $d^{\prime}$ & $\mathrm{RT}$ \\
\hline A. Performance & & & & & & \\
$\quad$ BAS group & & & & & & \\
$\quad$ High & 69 & 2.64 & 1,040 & 5 & 2.44 & 1,089 \\
$\quad$ Low & 60 & 2.34 & 1,038 & 9 & 2.09 & 1,134 \\
$\quad$ BIS group & & & & & & \\
$\quad$ High & 72 & 2.49 & 1,039 & 6 & 2.00 & 1,080 \\
$\quad$ Low & 57 & 2.53 & 1,038 & 8 & 2.38 & 1,147 \\
B. Effect sizes $(r)$ & & & & & & \\
$\quad$ BAS group & & $.27 *$ & .01 & & .27 & .09 \\
$\quad$ BIS group & & .06 & .04 & & .30 & .14 \\
\hline
\end{tabular}

Note-(A) Mean $d^{\prime}$ and response time (RT) are given for each personality group; (B) effect size $r$ s for the corresponding group effect (high vs. low). For the larger sample (which includes the smaller one), effect sizes were calculated using the $F$ value of the personality group effect from ANOVAs: BAS or BIS group (high, low) and stimulus type (word, face), between subjects. For the smaller sample, the effect sizes for personality group were calculated using $F$ values from ANOVAs: BAS or BIS group (high, low) between subjects. BAS, behavioral approach sensitivity; BIS, behavioral inhibition sensitivity. ${ }^{*} p=.0025$; for all other effects, $p s>.29$. 


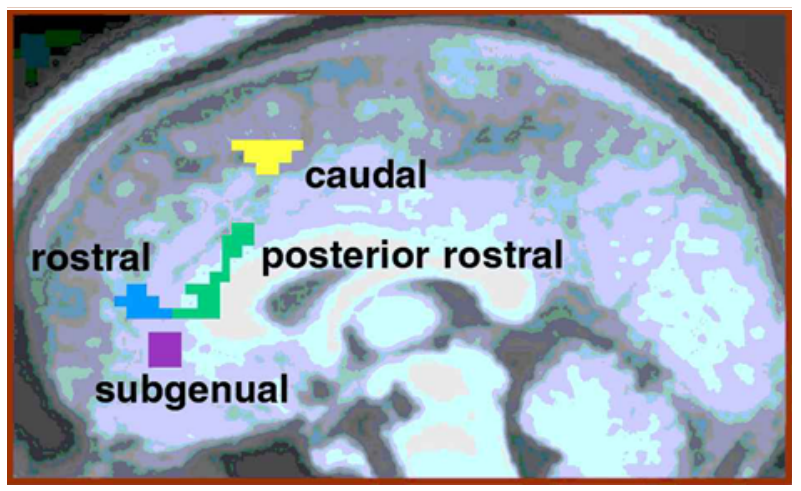

Figure 1. Regions of interest (ROIs) within the anterior cingulate cortex (ACC), shown in a medial sagittal view (anterior on the left, superior at top) at $X=-1.5$. Yellow $=$ caudal ACC (110 contiguous voxels at $-2,9,45)$; green $=$ posterior-rostral $\mathrm{ACC}(47$ contiguous voxels at $-2,24,12)$; blue $=$ rostral $\mathrm{ACC}(83$ contiguous voxels at $2,42,9)$; purple $=$ subgenual ACC $(26$ contiguous voxels at $2,36,-6)$. The ROIs were defined using functional criteria (see the text) and are superimposed on an average anatomical image from the same participants.

BAS group $[F(1,125)=9.49, p=.0025$; effect size, $r=$ .27], with no interaction between BAS group and stimulus type $[F(1,125)<0.01, p>.98]$. For RT, there was no effect of BAS group and no interaction between BAS group and stimulus type $\left[F_{\mathrm{s}}(1,125)<.75, p \mathrm{~s}>.38\right]$. For BIS, the high and low groups did not differ reliably on $d^{\prime}$ or RT $\left[F_{\mathrm{s}}(1,125)<0.6, p \mathrm{~s}>.4\right]$, nor were there interactions of BIS group with stimulus type for $d^{\prime}$ or RT $\left[F_{\mathrm{s}}(1,125)<\right.$ $1.7, p \mathrm{~s}>$.19]. Similarly, simple correlations suggested a weak but reliable relation only for BAS and $d^{\prime}[r(127)=$ $.18, p=.038]$, for BIS and $d^{\prime}[r(127)=-.03]$, and for $\mathrm{RT}$ and BAS or BIS $[r \mathrm{~s}(127)=.04$, all $p \mathrm{~s}>.4]$.

When these analyses were restricted to the 14 participants who were scanned (see Table 1), none of the effects was statistically significant $(p s>.29)$. Nonetheless, in terms of effect size, the same relation held between BAS and $d^{\prime}$ in those who were scanned as in the large sample (effect size, $r=.27$ ). The predicted direction of the effect held for BIS and $d^{\prime}(r=.30)$.

\section{Imaging Measures}

A premise of the investigation was that the relevant subregions of the ACC could be defined for these participants without reference to personality. To do so, we drew upon the large literature concerning the functional neuroanatomy of the ACC (for meta-analyses, see Bush et al., 2000; Devinsky et al., 1995; Paus et al., 1998; Shulman et al., 1997). To define regions of interest (ROIs) for subsequent analysis, we used the data from the two emotionally neutral conditions. We expected and found significant taskrelated activation in the caudal $A C C^{1}$ (i.e., more activity during task than during fixation for each voxel, $p<.001$ ). We expected and found deactivation (i.e., more activity during fixation than during task) in the rostral $A C C$ and the posterior-rostral ACC $(p<.001$ for each voxel), as well as in the subgenual ACC ( $p<.05$ for each voxel). The locations of the four ROIs are illustrated in Figure 1. Statistical analyses involving BIS and BAS were then computed on the MR signal extracted from and averaged across the voxels within each ROI.

Personality effects. To test whether personality was related to cognitive activation in the ACC, we conducted multiple regression analyses having the dependent variable of task-related activity averaged across the six experimental conditions. To control for resting state activity, average activity during fixation was first entered into the regression. To assess whether the BIS and BAS scores explained additional variance in the task-related activity, the partial correlation ( $p r$ ) of personality scores was then computed, controlling for fixation-related activity. Analyses are reported for BIS while controlling (via regression) for the possible influence of BAS and for BAS while controlling for BIS. This procedure was repeated for each of the four subregions of the ACC.

BAS scores predicted cognitive activation in the ACC (see Table 2). That is, the association of BAS with average ACC activity was significant when the demand for cognitive control was high (during task), controlling for low (during fixation). Of note, this held in the caudal (cognitive) area of the ACC ( $p r=-.84, p=.0012)$. It held weakly in the posterior-rostral ACC and, surprisingly, little if at all in the rostral and subgenual ACC. That is, high BAS participants had systematically smaller task-related increases from baseline, as was expected. The scatterplot in Figure 2 illustrates the relation between BAS and task activity in terms of percentage signal change (simple correlation, $r=-.81, p=.0003$ ). Importantly, the partial correlations for BAS were unchanged when we further explicitly controlled for behavioral performance (i.e., in addition to controlling for fixation-related activity and BIS), for both $d^{\prime}$ and RT ( $\left.p r s=-.83,-.84\right)$. The partial correlations for BAS were also unchanged when we explicitly controlled for emotional state, using the ratings of amused, anxious, energetic, or calm (average ratings across all scans; $p r s=-.84$ to -.87 ). In the caudal and posteriorrostral ACC, a relation held marginally between BIS and average ACC activity in the direction expected $(p r \mathrm{~s}=.55$,

Table 2

Personality Predicts Task-Related Anterior Cingulate Cortex (ACC) Activity Averaged Across Conditions

\begin{tabular}{lccccc}
\hline & \multicolumn{2}{c}{ Cognitive ROIs } & & \multicolumn{2}{c}{ Affective ROIs } \\
\cline { 2 - 3 } \cline { 5 - 6 } Group & Caudal & Posterior-Rostral & & Rostral & Subgenual \\
\hline BAS & $-.84^{*}$ & $-.68^{*}$ & & -.06 & .31 \\
BIS & .55 & .56 & & .06 & -.43 \\
\hline
\end{tabular}

Note-Each value in the table is a partial correlation of one personality variable with task-related ACC activity, as found by a hierarchical regression. For each regression, the dependent variable was task-related activity averaged across the six scans ( $n=13$ participants). Two regressions were carried out separately for each region of interest (ROI): one for BAS and one for BIS. The partial correlations for BAS were obtained by controlling for fixation and BIS. The partial correlations for BIS were obtained by controlling for fixation and BAS. BAS, behavioral approach sensitivity; BIS, behavioral inhibition sensitivity $*_{p}<.05$. 


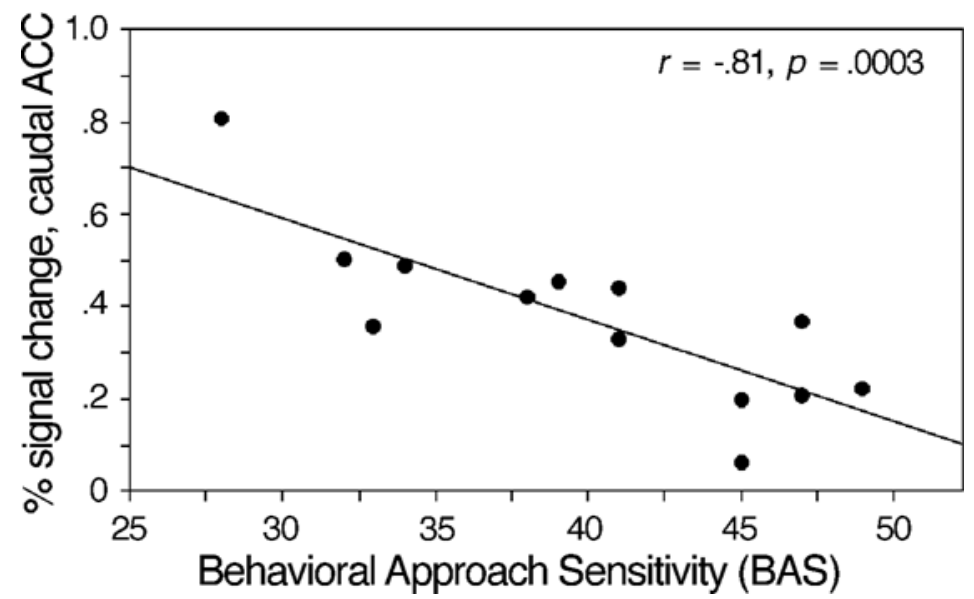

Figure 2. Self-reported personality (behavioral approach sensitivity, BAS) predicts task-related neural activity in the caudal anterior cingulate cortex (ACC). The dependent variable is the percentage of signal change during the three-back task from a fixation baseline. For each participant, percentage of signal change in the caudal ACC was averaged across the six scans (as in related analyses reported in Table 2).

$.56, p s<.10)$. That is, high-BIS participants had marginally larger increases in the caudal ACC, perhaps suggesting low statistical power rather than no effect.

The factorial design allowed us to isolate the potential influence of different emotional states on the personalityACC relationship. For each of the three emotion conditions (pleasant, neutral, unpleasant), we averaged the MR signal from the face and the word task conditions. Using the same regression procedure, we assessed whether task-related activity (restricted to a specific emotion condition) was predicted by personality scores within each emotion condition, after controlling for fixation-related activity. These analyses are summarized in Table 3. Within each emotion condition in isolation, the same relationship held between BAS and caudal ACC activity as held for all conditions averaged together. For BIS, the relationship held significantly in the pleasant emotion condition in the expected direction ( $p r=$ .82 ), but did not hold in the neutral or the unpleasant condition.

Emotional state. Emotional states also influenced ACC activity, by moderating the personality-ACC associations. There were no main effects of emotional state on taskrelated signal change in the $\operatorname{ACC}[F(2,24)=1.54, p=$ .23; main effects did hold in the lateral prefrontal cortex; see J. R. Gray et al., in press). Rather, in the ACC, emotional states appeared to strengthen the association between personality and task-related activity. We again used the same hierarchical regression strategy, here to assess the relation between personality (BIS, BAS) and activation in an emotional condition (pleasant, unpleasant) while controlling for both fixation-related activity and task-related activity in the neutral condition. In other words, in addition to controlling for fixation-related activity in the emotional condition, as was done previously, we further controlled for fixation-and task-related activity in the neutral conditions. These analyses are summarized in Table 4.

For the caudal ACC, personality scores explained variance in the task-related activity during the emotion conditions that was not explained by activity in the neutral condition. This relation held significantly for BAS in the unpleasant condition and for BIS in the pleasant condition. These analyses suggest that emotional state moderated the relationship between personality and taskrelated ACC activity. The relations between task-related ACC activity and BAS in the pleasant condition and task-related activity and BIS in the unpleasant condition had notable effect sizes, but without reaching significance. Inspection of the scatterplots showed that the emotion inductions led to both steeper regression slopes and tighter clustering of points around the regression lines, suggesting that emotional states lead to a stronger relation (within sub-

Table 3

Personality Predicts Task-Related Anterior Cingulate Cortex (ACC) Activity Within All Three Emotion Conditions Separately

\begin{tabular}{cccccc}
\hline & \multicolumn{2}{c}{ Cognitive ROIs } & & \multicolumn{2}{c}{ Affective ROIs } \\
\cline { 2 - 3 } \cline { 5 - 6 } Condition & Caudal & Posterior-Rostral & & Rostral & Subgenual \\
\hline Pleasant & & & & & \\
BAS & $-.79^{*}$ & -.44 & & -.22 & .13 \\
BIS & $.82^{*}$ & .19 & & .04 & -.29 \\
Neutral & & & & .37 & .39 \\
BAS & $-.63^{*}$ & -.20 & & .04 & -.52 \\
BIS & .36 & .43 & & .04 & \\
Unpleasant & & & & -.39 & .40 \\
BAS & $-.87^{*}$ & -.56 & & .12 & -.35 \\
BIS & -.22 & .31 & & .12 \\
\hline
\end{tabular}

Note-Same conventions as Table 2, with $n=14$ in pleasant and neutral conditions, $n=13$ in unpleasant condition. ${ }^{*} p<.05$. 
Table 4

Personality Predicts Task-Related Anterior Cingulate Cortex (ACC) Activity in the Emotion Conditions Using Multiple Regression to Control for Neutral Condition Activity

\begin{tabular}{lccccc}
\hline & \multicolumn{2}{c}{ Cognitive ROIs } & & \multicolumn{2}{c}{ Affective ROIs } \\
\cline { 2 - 3 } \cline { 5 - 6 } Condition & Caudal & Posterior-Rostral & & Rostral & Subgenual \\
\hline Pleasant & & & & & \\
BAS & -.49 & -.46 & & -.12 & -.15 \\
BIS & $.69 *$ & .24 & & .04 & -.02 \\
Unpleasant & & & & -.54 & .15 \\
BAS & $-.76 *$ & -.59 & & .19 & .00 \\
BIS & -.51 & .32 & & .19 \\
\hline
\end{tabular}

Note-Same conventions as Table 2, showing prs from regressions controlling for the emotion-fixation, neutral-task, and neutral-fixation conditions (in addition to controlling for the behavioral inhibition system (BIS) when examining the behavioral approach system (BAS), and BAS when examining BIS). $* p<.05$.

ject) and a more uniform relation (between subjects) between personality and ACC activity.

\section{DISCUSSION}

In terms of behavioral performance, high-BAS participants were more accurate than low-BAS participants, replicating previous work (Lieberman \& Rosenthal, 2001). We extended previous work by confirming that the same effect holds when measures of BAS are used and that the stimulus domain (verbal, nonverbal) had virtually no influence on the trait relation. We did not find a behavioral effect for BIS, possibly because we made no attempt to induce performance anxiety-for example, by introducing an ego threat that can be critical for eliciting performance decrements in trait anxious individuals.

In terms of brain activity, personality predicted taskrelated activation in the caudal (cognitive) ACC during a WM task. For BAS, this relation held consistently (1) for activity averaged across the three emotion inductions, (2) for the average activity, controlling for differences in behavioral performance and self-reported emotional states, (3) during three different manipulated emotional states separately, and (4) for the (unpleasant) emotional condition, controlling for activity in the neutral condition. For BIS, these same relations were mostly in the predicted directions, albeit they were only marginally significant and not consistent across conditions. This may be related to the lack of a reliable behavioral effect of BIS in our paradigm.

The relations of personality and brain activity were not attributable to differences in either behavioral performance or emotional state. There were no significant behavioral effects in the participants who were scanned, and the regression analyses specifically showed that any residual effects were incapable of explaining differences in brain activity. The absence of a significant BAS-performance relation in the participants who were scanned does not raise problems, because the effect size was the same as in the large sample. Because the caudal ACC is clearly involved in cognitive control, the null behavioral result in those who were scanned is methodologically advantageous. It argues against nonspecific accounts-for example, personality differences in the willingness to sustain an attempt at a difficult cognitive task, which would have influenced both performance and ACC activity (with differences in one covarying with differences in the other). Thus, a significant behavioral effect in the participants who were scanned could have been a relatively serious confound (although not necessarily a fatal one, in that multiple regression could still be used to identify any variance in ACC activity that did covary with personality but did not covary with performance).

The lack of a significant relation between personality and performance in those who were scanned may appear to leave open an alternative interpretation: The possibility that the association of BAS with ACC activity is due to individual differences in neural activity during fixation (resting state), rather than during task-related processing. This account is hard to completely rule out, although two aspects of the data argue against it. First, the behavioral relation between BAS and WM performance in the large sample was significant, in the predicted direction, and in line with previous work. Thus, a reliable relation held between BAS and behavioral performance, a relation that seems highly unlikely to depend on brain activity during a resting state. Critically, the same BAS-performance effect held for the participants who were scanned, as indexed by effect size. This, in turn, suggests that the relation between ACC activation and BAS was driven by task-related activity, and not by resting state (baseline) activity. A second argument against the alternative account is that it requires an association between resting state activity and personality in the caudal ACC specifically (because the present effect held in the caudal ACC specifically). However, resting state activity in the caudal ACC appears to have no relation to personality, based on two neuroimaging studies with large samples (Johnson et al., 1999; Zald et al., 2002); both of these did report an association for the rostral-subgenual ACC. Thus, an alternative account based on a personalityresting-state association is implausible for the caudal ACC. It may well partially explain the lack of reliable effects in the rostral-subgenual ACC.

In the analyses of emotional states as moderators of the personality-cognition relations, it is not obvious why the BAS relation was significant in the unpleasant states and the BIS relation in the pleasant states. We are reluctant to further interpret this aspect of the results, however, because it is quite possible that the effect size for BAS in the pleasant state $(-.49)$ and for BIS in the unpleasant state $(-.51)$ did not reach significance simply owing to low power ( $d f=8$ in these analyses). Although the associations that did hold are perhaps surprising, there is some precedent, in that extraversion predicted physiological responses to induced anxiety or stress (Kirschbaum et al., 1995). Regardless, induced emotional states influenced activity in the caudal ACC.

Overall, the data suggest (1) that personality differences in BAS are associated with differences in cognitive con- 
trol and (2) that the caudal ACC is influenced by both personality and induced emotion. The caudal ACC is part of a larger neural network involved in cognitive control, and its activity should be considered in this context. Our interpretation is that at least one component of the wider cognitive control network depends on BAS in a true functional sense. The caudal ACC could be that component or might simply come to reflect the activity of that component. The present data cannot discriminate between these alternatives. The data also imply that the network is sensitive to effects of emotional state (cf. J. R. Gray, 2001 ; J. R. Gray et al., in press), in that the emotion conditions moderated the relationships between personality and ACC activity. From this perspective, we discuss two theoretical implications.

\section{Implications for Personality}

To review the logic, previous work has suggested that two affective dimensions of personality are associated with individual differences in WM function. Consequently, these two traits should also be associated with individual differences in neural activity in the caudal ACC during a WM task. Individual differences in approach (BAS) and withdrawal (BIS) sensitivity should be the particularly relevant aspects of personality, based on the argument that BAS and BIS reflect stable differences in two fundamental behavioral control systems (Carver et al., 2000). Thus, we expected higher BAS to be associated with better WM performance and lower activation in the ACC and higher BIS to be associated with worse WM performance and higher activity. These predictions were upheld well for BAS and less well, if at all, for BIS.

The BAS result supports theories of personality in which differences in cognitive control are held to be important (Carver et al., 2000; Humphreys \& Revelle, 1984; Lieberman \& Rosenthal, 2001; Revelle, 1993). For example, Carver and colleagues argued for a conceptual integration of personality and action control, based on appetitive-aversive motivational systems and cybernetic control theory. Revelle has argued that "personality effects [on cognition] can be understood in terms of the differences in the way and in the rate at which parameters of the cognitive control system are adjusted to cope with changes in a constantly varying world" (p. 346). Such personality effects might be attributable to stable individual differences in physiological state parameters that are related to personality (e.g., arousal level, H. J. Eysenck, 1967; Humphreys \& Revelle, 1984; Lieberman \& Rosenthal, 2001; Revelle, 1993). Several neuroimaging studies have explicitly tested the hypothesis that trait differences in arousal should be revealed in resting state activity (e.g., Mathew, Weinman, \& Barr, 1984; Stenberg, Wendt, \& Risberg, 1993). The present data suggest that personality is associated not only with differences in resting brain activity (e.g., Ebmeier et al., 1994; Johnson et al., 1999; Sutton \& Davidson, 1997; Zald et al., 2002) or emotional reactivity (Canli et al., 2001; Costa \& McCrae, 1980; Larsen \& Ketelaar, 1991), but also with differences in cognitive reactivity. For BAS, the trait relations held in analyses partialling out emotional state and held consistently under divergent manipulated states (Table 3 ). This suggests that personality might be directly related to differences in cognitive control, not just indirectly via an influence of affective states associated with trait differences.

The present data suggest that the processing efficiency hypothesis for trait anxiety (M. W. Eysenck \& Calvo, 1992) should be extended to BAS. For trait anxiety, individual differences in cognitive control are not always evident in measures of behavioral performance and yet should be evident physiologically (M. W. Eysenck \& Calvo, 1992). For BAS, we found this key pattern of behavioral performance and brain activity: significant physiological differences that were not explained by behavioral differences. That is, high-BAS individuals may be more mentally efficient during WM tasks, as was shown by lower caudal ACC activity in those who were scanned. They may not always show a behavioral advantage, particularly if lowBAS individuals can compensate by maintaining an internal cognitive equilibrium in which caudal ACC activity is higher. Functional imaging can give access to internal constructs, such as compensation and effort, that are not easily assessed with external, global measures, such as behavioral performance.

The BAS-ACC relation may not generalize to extraversion in the broadest sense, as used by diverse theorists. Rather, on the basis of Carver et al.'s (2000) theoretical analysis, we expect it to hold for conceptions of extraversion (or subfactors) that emphasize individual differences in appetitive (approach-related) motivational systems.

\section{Implications for Theories of ACC Function}

Self-reported personality variables predicted cognitive task-related activity in the caudal ACC. This held particularly during emotional states, which might be expected to provoke or otherwise magnify affective trait differences in information processing. These data suggest that affective factors may be required for a complete understanding of the caudal (cognitive) subregions of the ACC. Numerous studies uphold a segregation of cognitive and affective processing in the ACC (Bush et al., 2000), and we agree with this distinction as a general characterization. However, our data suggest that, at a fine-grained level of analysis, a hard distinction between emotion and cognition may not be tenable, and so such a characterization of ACC may not be tenable. The data suggest that both affective personality traits and emotional states influence the cognitive control system and that the activity of the caudal ACC is sensitive to these affective influences. For this reason, cognitive activation studies of the caudal ACC may benefit from taking into account the emotional states and traits of the participants. This may be important whenever small samples are employed, particularly in between-subjects comparisons.

By focusing on ACC and using an $n$-back task, we sought to increase the probability of detecting an association be- 
tween ACC activity and personality variables. $N$-back tasks place high demands on cognitive control and robustly evoke neural activity within the caudal ACC and so were good tasks for present purposes. Although the $n$-back task is hardly a pure measure of cognitive control when contrasted with fixation, the basis of our claim of a trait relation with cognitive control is neuroanatomical:A great deal is known about the ACC. Activity in the caudal ACC is well established as indexing the demand for (or exertion of) control and is not correlated with personality under resting conditions. Another viable strategy would be to infer specificity on the basis of a task-type or trial-type comparison-that is, to compare brain activity during a cognitive process that requires control against activity during a process that is similar but does not require control and then to relate the control-specific neural activity to personality. Such a task comparison would isolate brain areas specific to cognitive control and would be strongly expected a priori to reveal activity in the caudal ACC.

Individual differences in cognitive control will need to be integrated theoretically with personality. Carver et al.'s (2000) action control hypothesis is clearly exciting, but control is discussed only in a very general sense (as discrepancy-reducing or discrepancy-enhancing feedback loops that promote approach toward a goal or withdrawal from an antigoal). Another possibility might be to extend the conflict-monitoring hypothesis of ACC function (Botvinick et al., 2001; Braver, Barch, Gray, Molfese, $\&$ Snyder, 2001; Carter et al., 1998; for a critique, see Bush et al., 2000). Under this hypothesis, the caudal ACC monitors for the occurrence of simultaneous activity in incompatible response pathways. Greater ACC activity indicates a high degree of response conflict and signals the need to engage control processes to resolve the conflict. This hypothesis might be generalizable to include emotional conflict (J. R. Gray \& Braver, in press). In theory, motivational salience and emotional conflict should vary as a function of individual differences in the sensitivity to cues of threat and reward-that is, BIS and BAS aspects of personality (cf. Dollard \& Miller, 1950; Miller, 1944). It is possible that a generalized conflict-monitoring hypothesis could serve as a united hypothesis for all the subregions of the ACC. Many manipulations that activate affective regions of the ACC (e.g., painful stimuli; Derbyshire et al., 1998) are likely to involve emotional conflict (i.e., conflict between voluntary endurance of pain vs. failure to comply with the experimental demands), not simply emotion. Many cognitive tasks that activate the caudal ACC (which tend to be difficult) also induce some degree of performance anxiety and subjective unpleasantness. In any case, our data clearly indicate that the caudal ACC is sensitive to integrated emotional and cognitive signals. How and why this occurs is an important research direction (Bush et al., 2000; Simpson et al., 2001).

To speculate, it is possible that the association of BAS and caudal ACC activity might be mediated by the neuromodulator dopamine. Extraverts have more reactive dopamine systems (Depue, Luciana, Arbisi, Collins, \& Leon,
1994), phasic dopamine bursts are released in response to unpredicted cues of reward (Schultz, Dayan, \& Montague, 1997), and ACC is a major recipient of dopamine projections (Williams \& Goldman-Rakic, 1993). An exploratory, whole-brain PET study of dopamine D2 receptor binding (Suhara et al., 2001) found a correlation in the anterior cingulate between $\mathrm{D} 2$ binding and the personality trait of novelty seeking (akin to extraversion and BAS; Cloninger et al., 1993; $r=-.45)$. Unfortunately, it was not reported whether this held in the caudal ACC, the rostral-subgenual ACC, or both. In other work, we have developed a computational model of cognitive control in which dopamine plays a critical role (Braver \& Cohen, 2000), based in part on considerable evidence for such interactions in human and nonhuman primates (e.g., Luciana, Depue, Arbisi, \& Leon, 1992; Sawaguchi \& Goldman-Rakic, 1991). Thus, it is possible that the dopamine system partially mediated the observed association between BAS and caudal ACC activity during the three-back task. A dopaminergic mechanism would also suggest another possible basis for a theoretical integration of personality and cognitive control: a relation between catecholamine systems and the construct of arousal, as currently used in the personality literature (Lieberman \& Rosenthal, 2001; Revelle, 1993).

Surprisingly, task-related activity in the rostral and subgenual (affective) ACC was not strongly related to personality. However, it would be inappropriate to conclude that affective regions are insensitive to personality-cognition relations. We may have lacked sufficient power to detect a weaker but real relation in these regions, especially for the subgenual ACC (based on the effect sizes; Tables 2 and 3). Alternatively, a relation may hold during resting conditions (Johnson et al., 1999; Zald et al., 2002) and be attenuated during cognitive conditions.

\section{REFERENCES}

BAdDELEY, A. D. (1986). Working memory. New York: Oxford University Press.

Bless, H., \& Schwarz, N. (1999). Sufficient and necessary conditions in dual-process models: The case of mood and information processing. In S. Chaiken \& Y. Trope (Eds.), Dual-process theories in social psychology (pp. 423-440). New York: Guilford.

Botvinick, M. M., Braver, T. S., Barch, D. M., Carter, C. S., \& CoHen, J. C. (2001). Conflict monitoring and cognitive control. Psychological Review, 108, 624-652.

Braver, T. S., Barch, D. M., Gray, J. R., Molfese, D. L., \& Snyder, A. (2001). Anterior cingulate cortex and response conflict: Effects of frequency, inhibition, and errors. Cerebral Cortex, 11, 825-836.

Braver, T. S., Barch, D. M., Kelley, W. M., Buckner, R. L., Cohen, N. J., Meizin, F. M., Snyder, A. Z, Olinger, J. M., Conturo, T. E., Akbudak, E., Conturo, T. E., \& Peterson, S. E. (2001). Direct comparison of prefrontal cortex regions engaged by working and long-term memory tasks. NeuroImage, 14, 48-59.

Braver, T. S., \& Cohen, J. D. (2000). On the control of control: The role of dopamine in regulating prefrontal function and working memory. In S. Monsell \& J. Driver (Eds.), Control of cognitive processes: Attention and performance XVIII (pp. 713-738). Cambridge, MA: MIT Press.

Braver, T. S., Cohen, J. D., Nystrom, L. E., Jonides, J., Smith, E. E., \& Noll, D. C. (1997). A parametric study of prefrontal cortex involvement in human working memory. NeuroImage, 5, 49-62.

Bush, G., LuU, P., \& Posner, M. I. (2000). Cognitive and emotional in- 
fluences in anterior cingulate cortex. Trends in Cognitive Sciences, $\mathbf{4}$, 215-222.

CABeza, R, \& Ny BERG, L. (2000). Imaging cognition: II. An empirical review of 275 PET and fMRI studies. Journal of Cognitive Neuroscience, 12, 1-47.

Canli, T., Zhao, Z., Kang, E., Gross, J., Desmond, J. E., \& Gabrieli, J. D. E. (2001). An $f$ MRI study of personality influences on brain reactivity to emotional stimuli. Behavioral Neuroscience, 115, 33-42.

Carter, C. S., Botvinick, M. M., \& Cohen, J. D. (2000). The contribution of anterior cingulate cortex to executive processes in cognition. Reviews in Neuroscience, 10, 49-57.

Carter, C. S., Braver, T. S., Barch, D. M., Botvinick, M. M., Noll, D. C., \& Cohen, J. D. (1998). Anterior cingulate cortex, error detection, and the online monitoring of performance. Science, 280, 747-749.

Carver, C. S., Sutton, S. K., \& Scheier, M. F. (2000). Action, emotion, and personality: Emerging conceptual integration. Personality \& Social Psychology Bulletin, 26, 741-751.

CArver, C. S., \& White, T. (1994). Behavioral inhibition, behavioral activation, and affective responses to impending reward and punishment: The BIS/BAS scales. Journal of Personality \& Social Psychology, 67, 319-333.

Cloninger, C. R., Svrakic, D. M., \& Przybeck, T. R. (1993). A psychobiological model of temperament and character. Archives of General Psychiatry, 50, 975-990.

Cohen, J., MacWhinney, B., Flatt, M., \& Provost, J. (1993). PsyScope: An interactive graphic system for designing and controlling experiments in the psychology laboratory using Macintosh computers. Behavioral Research Methods, Instruments, \& Computers, 25, 257-271.

Conturo, T. E., McKinst ry, R. C., Akbudak, E., Snyder, A. Z, Yang, T., \& Raichle, M. E. (1996, November). Sensitivity optimization and experimental design in functional magnetic resonance imaging. Paper presented at the Society for Neuroscience annual meeting, Washington, DC.

Costa, P. T., \& McCrae, R. R. (1980). Influence of extraversion and neuroticism on subjective well-being: Happy and unhappy people. Journal of Personality \& Social Psychology, 38, 668-678.

Damasio, A. R. (1994). Descartes' error: Emotion, reason and the human brain. New York: Grosset/Putnam.

DARKE, S. (1988). Effects of anxiety on inferential reasoning task performance. Journal of Personality \& Social Psychology, 55, 499-505.

DAVIDSON, R. J. (1995). Cerebral asymmetry, emotion, and affective style. In R. J. Davidson \& K. Hugdahl(Eds.), Brain asymmetry (pp.361-387). Cambridge, MA: MIT Press.

Depue, R. A., \& Collins, P. F. (1999). Neurobiology of the structure of personality: Dopamine, facilitation of incentive motivation, and extraversion. Behavioral \& Brain Sciences, 22, 491-569.

Depue, R. A., Luciana, M., Arbisi, P., Collins, P., \& Leon, A. (1994). Dopamine, and the structure of personality: Relation of agonist-induced dopamine activity to positive emotionality. Journal of Personality \& Social Psychology, 67, 485-498.

Derbyshire, S. W. G., Vogt, B. A., \& Jones, A. K. P. (1998). Pain and Stroop interference tasks activate separate processing modules in anterior cingulate cortex. Experimental Brain Research, 118, 52-60.

D'Esposito, M., Detre, J. A., Alsop, D. C., Shin, R. K., Atlas, S., \& Grossman, M. (1995, November 16). The neural basis of the central executive system of working memory. Nature, 378, 279-281.

Devinsky, O., Morrell, M. J., \& Vogt, B. (1995). Contributions of anterior cingulate cortex to behavior. Brain, 118, 279-306.

Dollard, J., \& Miller, N. E. (1950). The dynamics of conflict: Their implications for therapy. In J. Dollard \& N. E. Miller (Eds.), Personality and psychotherapy (pp. 352-368). New York: McGraw-Hill.

Drevets, W. C., Price, J. L., Simpson, J. R., Jr., Todd, R. D., Reich, T., VAnnier, M., \& Raichle, M. E. (1997). Subgenual prefrontal cortex abnormalities in mood disorders. Nature, 386, 824-827.

Duncan, J., Seitz, R. J., Kolodny, J., Bor, D., Herzog, H., Ahmed, A., Newell, F. N., \& EMSLIE, H. (2000). A neural basis for general intelligence. Science, 289, 457-460.

EAsterbrooK, J. A. (1959). The effect of emotion on cue utilization and the organization of behavior. Psychological Review, 66, 183-201.

Ebmeier, K. P., Deary, I. J., O’Carroll, R. E., Prentice, N., MofFOot, A. P. R. \& Goodwin, G. M. (1994). Personality associations with the uptake of the cerebral blood flow marker 99mTc-Exametazime estimated with single photon emission tomography. Personality \& Individual Differences, 17, 587-595.

EYSENCK, H. J. (1967). The biological basis of personality. Springfield, IL: Thomas.

Eysenck, H. J., \& Eysenck, M. W. (1985). Personality and individual differences: A natural science approach. New York: Plenum.

EYSENCK, M. W. (1979). Anxiety, learning, and memory: A reconceptualization. Journal of Research in Personality, 13, 363-385.

EysencK, M. W., \& CALVo, M. G. (1992). Anxiety and performance: The processing efficiency theory. Cognition \& Emotion, 6, 409-434.

Ey SENCK, S. B. G., Ey SENCK, H. J., \& BARRETt, P. (1985). A revised version of the psychoticism scale. Personality \& Individual Differences, 6, 21-29.

Funder, D. C. (2001). Personality. Annual Review of Psychology, 52, 197-221.

GoldMAN-RAKIC, P. S. (1987). Circuitry of primate prefrontal cortex and regulation of behavior by representational memory. In F. Plum \& V. Mountcastle (Eds.), Handbook of physiology: The nervous system (Vol. 5, pp. 373-417). Bethesda, MD: American Physiological Society.

GraY, J. A. (1970). The psychophysiological basis of introversionextraversion. Behavioural Research \& Therapy, 8, 249-266.

GRAY, J. A. (1982). The neuropsychologyof anxiety: An inquiry into the functions of the septo-hippocampal systems. Oxford: Oxford University Press.

Gray, J. A. (1994). Personality dimensions and emotion systems. In P. Ekman \& R. J. Davidson (Eds.), The nature of emotion (pp. 329331). New York: Oxford University Press.

GRAY,J. R. (2001). Emotional modulation of cognitive control: Approachwithdrawal states double-dissociate spatial from verbal two-back task performance. Journal of Experimental Psychology: General, 130, 436-452.

GraY, J. R., \& BraVER, T. S. (in press). Integration of emotion and cognitive control: A neurocomputational hypothesis of dynamic goal regulation. In S. C. Moore \& M. R. Oaksford (Eds.), Emotional cognition. Amsterdam: John Benjamins.

Gray, J. R., Braver, T. S., \& Raichle, M. E. (in press). Integration of emotion and cognition in lateral prefrontal cortex. Proceedings of the National Academy of Sciences.

Gross, J., Sutton, S. K., \& KetelaAr, T. (1998). Relations between affect and personality: Support for the affect-level and affect-reactivity views. Personality \& Social Psychology Bulletin, 24, 279-288.

Harmon-Jones, E., \& Allen, J. J. (1997). Behavioral activation sensitivity and resting frontal EEG asymmetry: Covariation of putative indicators related to risk for mood disorders. Journal of Abnormal Psychology, 106, 159-163.

Harmon-Jones, E. \& Sigelman, J. (2001). State anger and prefrontal brain activity: Evidence that insult-related relative left-prefrontal activation is associated with experienced anger and aggression. Journal of Personality \& Social Psychology, 80, 797-803.

Humphreys, M. S., \& Revelle, W. (1984). Personality, motivation, and performance: A theory of the relationship between individual differences and information processing. Psychological Review, 91, 153-184.

IsEN, A. M. (1993). Positive affect and decision making. In M. Lewis \& J. Haviland (Eds.), Handbook of emotion (pp. 261-277). New York: Guilford.

Johansen, J. P., Fields, H. L., \& Manning, B. H. (2001). The affective component of pain in rodents: Direct evidence for a contribution of the anterior cingulate cortex. Proceedings of the National Academy of Sciences, 98, 8077-8082.

Johnson, D. L., Wiebe, J. S., Gold, S. M., Andreasen, N. C., Hichwa, R. D., Watkins, G. L., \& Boles Ponto, L. L. (1999). Cerebral blood flow and personality: A positron emission tomography study. American Journal of Psychiatry, 156, 252-257.

Kirschbaum, C., Prussner, J. C., Stone, A. A., Federenko, I., Gaab, J., Lintz, D., Schommer, N., \& Hellhammer, D. H. (1995). Persistent high cortisol responses to repeated psychological stress in a subpopulation of healthy men. Psychosomatic Medicine, 57, 468-474.

Larsen, R. J., \& Buss, D. M. (2002). Personality psychology. New York: McGraw-Hill.

Larsen, R. J., \& Ketelaar, E. (1991). Personality and susceptibility to 
positive and negative emotional states. Journal of Personality \& Social Psychology, 61, 132-140.

Leon, M. R., \& Revelle, W. (1985). Effects of anxiety on analogical reasoning: A test of three theoretical models. Journal of Personality \& Social Psychology, 49, 1302-1315.

Lieberman, M. D. (2000). Introversion and working memory: Central executive differences. Personality \& Individual Differences, 28, 479-486.

Lieberman, M. D., \& Rosenthal, R. (2001). Why introverts can't always tell who likes them: Multitasking and nonverbal decoding. Journal of Personality \& Social Psychology, 80, 294-310.

Luciana, M., Depue, R. A, Arbisi, P., \& Leon, A. (1992). Facilitation of working memory in humans by a $\mathrm{D}_{2}$ dopamine receptor agonist. Journal of Cognitive Neuroscience, 4, 58-68.

LuU, P., Colins, P., \& Tucker, D. M. (2000). Mood, personality, and selfmonitoring: Negative affect and emotionality in relation to frontal lobe mechanisms of error monitoring. Journal of Experimental Psychology: General, 129, 43-60.

Luu, P., Tucker, D. M., Derryberry, D., Reed, M., \& Poulsen, C. (in press). Electrophysiological responses to errors and feedback. Psychological Science.

Mathew, R. J., Weinman, M. L., \& Barr, D. L. (1984). Personality and regional cerebral blood flow. British Journal of Psychiatry, 144, 529-532.

Mathews, G., Davies, R., \& LeEs, J. L. (1990). Arousal, extraversion, and individual differences in resource availability. Journal of Personality \& Social Psychology, 59, 150-168.

Mayberg,H. S., Brannan, S. K., Mahurin, R. K., Jerabek, P. A., Brickman, J. S., Tekell, J. L., Silva, J. A. McGinnis, S., Glass, T. G., MarTIN, C. C., \& Fox, P. T. (1997). Cingulate function in depression: A potential predictor of treatment response. NeuroReport, 8, 1057-1061.

Metcalfe, J., \& Mischel, W. (1999). A hot/cool-system analysis of delay of gratification: Dynamics of willpower. Psychological Review, 106, 3-19.

MiLler,N. E (1944). Experimental studies of conflict. In J. M. Hunt (Ed.), Personality and the behavior disorders (Vol. 1, pp. 431-465). New York: Ronald.

Miltner, W. H. R., Braun, C. H., \& Coles, M. G. H. (1997). Eventrelated brain potentials following incorrect feedback in a timeestimation task: Evidence for a "generic" neural system for error detection. Journal of Cognitive Neuroscience, 9, 788-798.

Miyake, A., \& Shah, P. (Eds.) (1999). Models of working memory: Mechanisms of active maintenance and executive control. New York: Cambridge University Press.

Mugler, J. P. I., \& Brookeman, J. R. (1990). Three-dimensional magnetization-prepared rapid gradient-echo imaging (3D MPRAGE). Magnetic Resonance in Medicine, 15, 152-157.

Paus, T., Koski, L., Caramanos, Z., \& Westbury, C. (1998). Regional differences in the effects of task difficulty and motor output on blood flow response in the human anterior cingulate cortex: A review of 107 PET activation studies. NeuroReport, 9, R37-R47.

Posner, M. I., \& DiGirolamo, G. J. (1998). Executive attention: Conflict, target detection and cognitive control. In R. Parasuraman (Ed.), The attentive brain (pp. 401-423). Cambridge, MA: MIT Press.

Posner, M. I., \& Petersen, S. E. (1990). The attention system of the human brain. Annual Review of Neuroscience, 13, 25-42.

REVELLE, W. (1993). Individual differences in personality and motivation: "Non-cognitive" determinants of cognitive performance. In A. Baddeley \& L. Weiskrantz (Eds.), Attention: Selection, awareness, and control (pp. 346-373). New York: Oxford.

SAWAGUCHI, T., \& GoldMAN-RAKIC, P. S. (1991). D1 dopamine receptors in prefrontal cortex: Involvement in working memory. Science, 251, 947-950.
Schmidt, L. A. (1999). Frontal brain electrical asymmetry in shyness and sociability. Psychological Science, 10, 316-320.

Schultz, W., Dayan, P., \& Montague, P. R. (1997). A neural substrate of prediction and reward. Science, 275, 1593-1599.

Shulman, G. L., Fiez, J. A., Corbetta, M., Buckner, R. L., Miezin, F. M., Raichle, M. E., \& Petersen, S. E. (1997). Common blood flow changes across visual tasks: II. Decreases in cerebral cortex. Journal of Cognitive Neuroscience, 9, 648-663.

Simon, H. A. (1967). Motivational and emotional controls of cognition. Psychological Review, 74, 29-39.

Simpson, J. R., Snyder, A. Z, Gusnard, D. A. \& Raichle, M. E. (2001). Emotion-induced changes in human medial prefrontal cortex: I. During cognitive task performance. Proceedings of the National Academy of Sciences, 98, 683-687.

SMith, E. E., \& Jonides, J. (1999). Storage and executive processes in the frontal lobes. Science, 283, 1657-1661.

Stenberg, G., Wendt, P. E., \& Risberg, J. (1993). Regional cerebral blood flow and extraversion. Personality \& Individual Differences, 15 , 547-554.

Suhara, T., Yasuno, F., Sudo, Y., Yamamoto, M., Inoue, M., OKubo, Y., \& SUZUKI, K. (2001). Dopamine $\mathrm{D}_{2}$ receptors in the insular cortex and the personality trait of novelty seeking. Neurolmage, 13, 891-895.

Sutton, S. K., \& DAvidson, R. J. (1997). Prefrontal brain asymmetry: A biological substrate of the behavioral approach and inhibition systems. Psychological Science, 8, 204-210.

TAlairach, J., \& Tournoux, P. (1988). Co-planar stereotaxic atlas of the human brain. New York: Thieme.

Watson, D., Clark, L. A., \& Tellegen, A. (1988). Developmental and validation of brief measures of positive and negative affect: The PANAS scales. Journal of Personality \& Social Psychology, 54, 1063-1070.

Watson, D., \& Tellegen, A. (1985). Toward a consensual structure of mood. Psychological Bulletin, 98, 219-235.

Whalen, P. J., Bush, G., McNally, R. J., Wilhelm, S., McInerney, S. C., Jenike, M. A., \& RaUCH, S. L. (1998). The emotional counting Stroop paradigm: A functional magnetic resonance imaging probe of the anterior cingulate affective division. Biological Psychiatry, 44, 1219-1228.

Williams, M. S., \& Goldman-RaKic, P. S. (1993). Characterization of the dopaminergic innervation of the primate frontal cortex using a dopamine-specific antibody. Cerebral Cortex, 3, 199-222.

Yerkes, R. M., \& Dodson, J. D. (1908). The relation of strength of stimulus to the rapidity of habit formation. Journal of Comparative Neurology \& Psychology, 18, 459-482.

Zald, D., Mattson, D. L., \& PARdo, J. V. (2002). Brain activity in ventromedial prefrontal cortex correlates with individual differences in negative affect. Proceedings of the National Academy of Sciences, 99 , 2450-2454.

ZeLENSKI, J. M., \& LARSEN, R. J. (1999). Susceptibility to affect: A comparison of three personality taxonomies. Journal of Personality, 67, 761-791.

\section{NOTE}

1. Activation in the caudal ACC was contiguous with activation in the supplementary motor area, as has commonly been observed. Given our interest in the ACC specifically, we retained voxels with $Z<50$ and $Y>0$ (see Bush et al., 2000).

(Manuscript received July 9, 2001; revision accepted for publication December 10, 2001.) 